\title{
Development of a new colorimetric assay for detection of bisphenol-A in aqueous media using green synthesized silver chloride nanoparticles: experimental and theoretical study
}

\author{
Shiva Khalililaghab ${ }^{1}$ - Safieh Momeni ${ }^{2}$ - Maryam Farrokhnia ${ }^{2}$ - Iraj Nabipour ${ }^{2}$. \\ Sadegh Karimi ${ }^{1}$
}

Received: 1 November 2016 /Revised: 13 January 2017 / Accepted: 26 January 2017 / Published online: 8 February 2017

(C) Springer-Verlag Berlin Heidelberg 2017

\begin{abstract}
In the present study, a cost-effective, green and simple synthesis method was applied for preparation of stable silver chloride nanoparticles (AgCl-NPs). The method was done by forming $\mathrm{AgCl}-\mathrm{NPs}$ from $\mathrm{Ag}^{+}$ions using aqueous extract of brown algae (Sargassum boveanum) obtained from the Persian Gulf Sea. This extract served as capping agent during the formation of AgCl-NPs. Creation of AgCl-NPs was confirmed by UV-visible spectroscopy, powder X-ray diffraction, energy-dispersive X-ray spectroscopy, and highresolution transmission electron microscopy, while the morphology and size analyses were characterized using highresolution transmission electron microscopy and dynamic light scattering. After optimization of some experimental conditions, particularly $\mathrm{pH}$, a simple and facile system was developed for the naked-eye detection of bisphenol-A. Moreover, a theoretical study of $\mathrm{AgCl}$ interaction with bisphenol-A was performed at the density functional level of theory in both gas and solvent phases. Theoretical results showed that electrostatic and van der Waal interactions play important roles in complexation of bisphenol-A with AgCl-NPs, which can lead to aggregation of the as-prepared $\mathrm{AgCl}-\mathrm{NPs}$ and results in color change from specific yellow to dark purple, where a
\end{abstract}

Electronic supplementary material The online version of this article (doi:10.1007/s00216-017-0230-0) contains supplementary material, which is available to authorized users.

Sadegh Karimi

karimi.sadegh@gmail.com; sakarimi@pgu.ac.ir

1 Department of Chemistry, College of Science, Persian Gulf University, Bushehr, Iran

2 The Persian Gulf Marine Biotechnology Research Center, The Persian Gulf Biomedical Sciences Research Institute, Bushehr University of Medical Sciences, Bushehr 7514763448, Iran new aggregation band induced at $542 \mathrm{~nm}$ appears. The absorbance at $542 \mathrm{~nm}$ was found to be linearly dependent on the bisphenol-A concentration in the range of $1 \times 10^{-6}-1 \times$ $10^{-4} \mathrm{M}$, with limit of detection of $45 \mathrm{nM}$. In conclusion, obtained results from the present study can open up an innovative application of the green synthesis of AgCl-NPs using brown algae extract as colorimetric sensors.

Keywords Silver chloride nanoparticle · Green synthesis · Bisphenol-A · Colorimetric detection · Brown algae $\cdot$ Density functional theory

\section{Introduction}

Metal nanoparticles (NPs) have attracted much attention due to their unique optical properties which originate from charge density oscillations [1,2]. These optical properties which are referred to as localized surface plasmon resonance (LSPR) have wide applications in bio-sensing [3], catalysis [4], cellular imaging [5], cancer clinical diagnosis [6], and cell targeting and therapy [7]. Hence, several strategies have been reported in the literature for preparation of nanoparticles comprising wet chemical approach [8], mechanical preparation [9], photochemical synthesis [10], and electrochemical methods [11]. However, the most commonly used procedure among the aforementioned ones is the wet chemical method, in which chemical reducing reagents are employed such as citrate [12, 13], hydroxylamine hydrochloride [14], and $\mathrm{NaBH}_{4}$ [15]. These conventional methods of nanoparticle synthesis have their own weakness and drawbacks. First of all, most of them are expensive and often produce unstable nanoparticles; hence, surfactants should be used [16]. Moreover, Murphy and Wyatt [17] indicate that using surfactant might introduce certain unfavorable cytotoxic effects. 
Furthermore, because of their difficult and time-consuming preparation, it is not possible to generalize them. Hence, in order to overcome these problems, especially there is growing concern about the hazards of the chemical-based nanoparticle production systems, the green chemistry-based synthesis of nanoparticle is considered as a better and eco-friendly approach for nanoparticle production [18-20]. Green synthesis can be done by biological process using bacteria, fungi, and algae which the latter one shows more advantages [21-23]. For example, the green synthesis by algae extract eliminates the cell culture maintaining process, and also, it is more suitable for large-scale production of metal nanoparticles [24]. In most of the green synthesis approaches, the nanoparticle is produced through redox reactions because natural sources like marine algae abundantly contain bio-compounds such as polysaccharides, phenolic compounds, and sugars which can act as reducing reagents in addition to serving as capping agent at the same time.

In the present work, simple and green procedure for synthesis of stable AgCl-NPs was used. In this procedure, $\mathrm{AgNO}_{3}$ was converted to AgCl-NPs using aqueous extract of Sargassum boveanum algae from Persian Gulf under neutral conditions. Furthermore, the colorimetric assay of synthesized nanoparticle was applied for detection of bisphenol-A (BPA) in tap and mineral water. The BPA was used as the detecting target because it is widely used for the manufacture of polycarbonate plastics (PCs), epoxy resins, and other plastics [25]. However, ester bonds that link the BPA monomers in these plastic or resin materials will be hydrolyzed, upon exposure to heat, acid, or base; consequently, BPA is released into the environment. Thus, the concerns about BPA have risen because this compound has estrogenic effect on animals at low doses. In addition, important subject is about high risk of breast cancer occurrence resulted from usage of BPA [26]. Thus, the great effort needs to develop simple and selective methods for determination of trace amounts of BPA [25]. Finally, theoretical study at the density functional level of theory was performed to elucidate the interactions between $\mathrm{AgCl}$ and BPA in both gas and solution phases. The results were applied to explain the preferred affinity of $\mathrm{AgCl}$ to $\mathrm{BPA}$ with respect to $\mathrm{Ag}$. To the best of our knowledge, this is the first colorimetric sensor application of silver chloride nanoparticles for analytical application.

\section{Experimental and theoretical section}

\section{Materials and reagents}

Brown algae (S. boveanum) were collected from the Persian Gulf in the south of Iran (Bushehr Province). Then, algae samples were washed with the fresh water followed by distillated water at least three times to remove the unwanted materials, and they were dried in the shadow for 1 week. The finely dried samples were ground and stored in refrigerator for further work. All the chemical reagents were of analytical grade and used directly without any further purifications. Also, they were stored at $4{ }^{\circ} \mathrm{C}$ in a refrigerator until use. Silver nitrate $\left(\mathrm{AgNO}_{3}\right), \mathrm{NaOH}$, hydrochloric acid $(\mathrm{HCl})$, bisphenol-A, phenol, chlorophenol, bromophenol, 1propanol, 2-butanole, ethyl acetate, ethyl benzoate, benzyl alcohol, phthalic acid, acetic acid, ethanol, methanol, $\mathrm{Cu}\left(\mathrm{NO}_{3}\right)_{2} \cdot 6 \mathrm{H}_{2} \mathrm{O}, \mathrm{Ni}\left(\mathrm{NO}_{3}\right)_{2} 6 \mathrm{H}_{2} \mathrm{O}, \mathrm{Pb}\left(\mathrm{NO}_{3}\right)_{2}, \mathrm{Fe}\left(\mathrm{NO}_{3}\right)_{3}$. $9 \mathrm{H}_{2} \mathrm{O}, \mathrm{Zn}\left(\mathrm{NO}_{3}\right)_{2} 6 \mathrm{H}_{2} \mathrm{O}, \mathrm{Co}\left(\mathrm{NO}_{3}\right)_{2} \cdot 6 \mathrm{H}_{2} \mathrm{O}, \mathrm{KNO}_{3}, \mathrm{NaF}$, $\mathrm{Na}_{2} \mathrm{SO}_{4}, \mathrm{Na}_{2} \mathrm{CO}_{3}$, and $\mathrm{Mn}\left(\mathrm{NO}_{3}\right)_{2} 5 \mathrm{H}_{2} \mathrm{O}$ were obtained from Sigma-Aldrich (St. Louis, MO, USA).

\section{Process of seaweed extraction}

The seaweed extraction process was simply done in two steps. At first step, $2 \mathrm{~g}$ of dry algae powder was taken along with $100 \mathrm{ml}$ of double-distillated water in conical flask. It was placed in an orbital shaker and boiled for $15 \mathrm{~min}$. Then, the crude extract was passed through a Whatman No.1 filter paper, and the filtrate sample was stored at $4{ }^{\circ} \mathrm{C}$ for further use.

\section{Instrumentation}

UV-vis absorption spectra were measured on Specord Analytic Jena UV-visible spectrophotometer. The morphology and size of synthesized nanoparticles were visualized by high-resolution transmission electron spectroscopy (HRTEM) on a Zeiss-EM10C-80 kV. X-ray diffraction measurements were carried out on a D8, Avance, Bruker, AXS diffractometer with $\mathrm{Cu}-\mathrm{K} \alpha$ radiation $(\lambda=1.5418 \AA)$ with a scan speed of $2 \% \mathrm{~min}$. The energy-dispersive X-ray spectroscopy (EDX) result was performed by an EDXA spectrometer in STEM mode. A dynamic light scattering (DLS) particle size analyzer (Horiba LB-550) was used to determine the distribution of the particle sizes. Fourier transform infrared (FTIR) spectroscopy measurement was adopted according to following procedure. The synthesized silver chloride nanoparticles by brown algae were centrifuged at $10,000 \mathrm{rpm}$ for $15 \mathrm{~min}$ and then dried, and its powder was subjected to FTIR spectroscopy measurement (Bruker Vector22 spectrometer) in the transmittance mode at a resolution of $4 \mathrm{~cm}^{-1}$ in $\mathrm{KBr}$ pellets. Also, the spectrum of a clean $\mathrm{KBr}$ disc (without AgCl-NPs) was used for background subtraction. Finally, all glass wares used in the following procedure were cleaned in a bath of freshly prepared 3:1 $(v / v) \mathrm{HCl}-$ $\mathrm{HNO}_{3}$, rinsed thoroughly in deionized water, and dried in oven. 


\section{Computational details}

The electronic study of all the investigated compounds was performed at the density functional theory (DFT) level. The B3lyp hybrid functional $[27,28]$ was applied to the systems. The 6-311++g** basis set was considered for main group atoms and LANL2DZ basis set for silver. Full geometry optimizations of all the molecules were carried out without symmetry constrain. The minima on the potential energy surfaces were qualified by the absence of negative eigenvalues in the diagonalized Hessian matrix, giving imaginary normal vibrational mode. Moreover, the molecular orbital (MO) study such as HOMO and LUMO energies was also performed. The NBO [29] and QTAIM [30] analyses were carried out on optimized structures to reveal the nature of interactions. Within the NBO analysis introduced by Weinhold and coworkers [31], we mainly paid particular attention to natural population analysis (NPA) charges and charge transfers [32]. The electron density and its Laplacian at the bond critical points (BCP) were computed based on Bader's QTAIM using the AIM2000 software [33]. The solvent effect on the electronic and structural properties in addition to intermolecular interaction energies were evaluated by employing the selfconsistent reaction field (SCRF) method with the polarized continuum model (PCM). Water was selected as a solvent for the study. All calculations were done by the Gaussian 09 suite of programs [34].

\section{Synthesis of silver chloride nanoparticles using Sargassum algae}

There are numerous ways to synthesis of AgNPs; nevertheless, only a few papers have reported for the preparation of $\mathrm{AgCl}-\mathrm{NPs}[22,35]$. Thus, we expected to obtain AgCl-NPs by brown algae extract according to Dhas [22] and Gopinath reports [35]. The brown algae extract contains several chemically important molecules such as polysaccharide (mainly alginate) and phenolic compounds; hence, they can act as stabilizing agent in green synthesis of metal nanoparticle. In the present study, the mixture (seaweed/ $/ \mathrm{gNO}_{3}$ ) was put into a rotary shaker at $80{ }^{\circ} \mathrm{C}(160 \mathrm{rpm})$ for $4 \mathrm{~h}$ and maintained in the dark. The progress of the reaction was monitored by observing color changes as well as recording UV-visible spectrum. The changes were found in the color of solution, which was from yellow solution turned to dark brown indicating the formation of nanoparticles.

In order to obtain the optimum volume ratio of seaweed to $\mathrm{AgNO}_{3}$, the aqueous algae extract was used and mixed with $1.0 \mathrm{mM} \mathrm{AgNO}_{3}$ with the volume ratio varying from 5:95 to 90:10 (see Electronic supplementary material (ESM) Fig. S1). Due to our main focus on sensing BPA, whole optimization procedure was done in the presence of this analyte. It should be mentioned that the concentration of BPA has been considered as $80 \mu \mathrm{M}$. Moreover, in each volume ratio, the $\mathrm{pH}$ condition was also investigated from $\mathrm{pH}=3$ to 11 , and the color change/UV-vis spectra of solution were followed. As we can see in ESM Fig. S1, when the volume ratio of seaweed $/ \mathrm{AgNO}_{3}$ decreased, the absorbance peak of obtained solution at $542 \mathrm{~nm}$ increased and reached to its maximum value at 5:95. Thus, we selected this ratio as optimum values of seaweed $/ \mathrm{AgNO}_{3}$ for further study. It is worthy of note that when crude extract volume is not high enough $(<5 \mathrm{ml})$, the formation of nanoparticle process does not take place favorably in the solution because of some reasons. First of all, the chlorine ion content of extract is not enough for $\mathrm{AgCl}$ creation particularly when less than $5 \mathrm{ml}$ of extract was used. Second, since the most part of the $\mathrm{Ag}^{+}$ions do not convert to $\mathrm{AgCl}$ in lower ratio of seaweed, they remain in the solution and this led to decrease the sensitivity of our proposed sensor. The third reason is related to the bio-extract components which are responsible for stabilization of the nanoparticles. When the ratio is lower than 5:95, our synthesis nanoparticles were less stable. Finally the time of nanoparticle synthesis was very longer at lower ratio, at least $18 \mathrm{~h}$ was needed. It should be noted that in the present study, $\Delta_{\text {absorbance }}\left(\mathrm{Abs}_{\text {sample }}-\mathrm{Abs}_{\mathrm{AgCl}-\mathrm{NPs}}\right)$ has been used for our study.

\section{Characterization of the as-prepared AgCl-NPs}

First of all, the formation of AgCl-NPs was qualitatively confirmed by color change (yellowish to dark brown) in $4 \mathrm{~h}$. Mulvaney [36] points out that the color change results from collective oscillation of free conduction electrons induced by an interacting electromagnetic field. Figure S2 (see ESM) indicates that the intense UV-vis spectrum peak of synthesized AgCl-NPs is centered at $417 \mathrm{~nm}$. In order to describe which functional groups in the marine extract are responsible for the stabilization of the silver chloride nanoparticles, the FTIR spectra of marine algae extract and silver chloride nanoparticles were investigated. As shown in ESM Fig. S3, a broad intense band at $3417 \mathrm{~cm}^{-1}$ in both spectra can be assigned to the $\mathrm{N}-\mathrm{H}$ stretching arising from the amino acid and peptides of protein and also $\mathrm{O}-\mathrm{H}$ of poly phenol compounds in aqueous extract [37]. The peak at $2927 \mathrm{~cm}^{-1}$ illustrates the asymmetric-CH stretching. Furthermore, the presence of peaks at 1659 and $1460 \mathrm{~cm}^{-1}$ confirms the amide I (CO symmetric stretching from amide bonds) and amide II (NH in-plane bend) groups of proteins [38] in both samples. There is a sharp band at $1024 \mathrm{~cm}^{-1}$ in the silver chloride nanoparticles which may be assigned as $\mathrm{C}-\mathrm{O}$ and $\mathrm{C}-\mathrm{C}$ stretching vibrations of pyranose ring common to all polysaccharides [39] of marine algae extract. These functional groups of biocompounds are mostly responsible for high stability of our synthesized silver chloride nanoparticles over months. 
High-resolution-TEM image discloses that AgCl-NPs were nearly spherical in the shape (Fig. 2a). Interestingly, the extracted bio-components of brown algae are also recognizable in this figure. These biocompound materials can bind to nanoparticle surface and play an important role to stabilization of the nanoparticle through several intermolecular interactions [22].

Dynamic light scattering (DLS) was employed to examine the size change of AgCl-NPs. The stabilized AgCl-NPs were dispersed in the solution and the size was about $65 \mathrm{~nm}$ (Fig. 2b). The crystallite structure of the biosynthesized AgCl-NPs was investigated by XRD analysis. This structure is shown in Fig. 2e; eight major peaks at 27.91, 32.31, 46.31, $54.91,57.25,67.3,74.12$, and 77.61 are illustrated on the $2 \theta$ scale and correspond to the (111), (200), (220), (311), (222), (400), (331), and (420), respectively [40]. These evidences confirm the formation of AgCl-NPs.

Energy-dispersive X-ray (EDX) analysis was performed in the parallel to verify the presence of elemental silver and chloride inside the green synthesized nanoparticles. In EDX analysis, two strong signals (silver and chloride) were observed (Fig. 2f). Consequently, although it is complicated to clearly disclose the mechanism of AgCl-NP formation due to complex media of crude extract, it sounds that the chlorine content of our brown algae extract can be source for the formation of AgCl-NPs in accordance with previous report [22]. Moreover, Gopinath et al. claimed that the chlorine ions of used extract, which we also confirmed their presence in the algae extract (ESM Fig. S4), react with the $\mathrm{AgNO}_{3}$ to form AgCl-NPs [35]. In other words, the possible mechanism for creation of such nanoparticles is similar to what Gopinath et al. [35] and Dhas [22] have been described.

\section{Result and discussion}

\section{Optimization of experimental condition}

Figure 1 illustrates the possible colorimetric sensing mechanism of BPA. As it is clear from this figure, BPA contains two hydroxyl $(-\mathrm{OH})$ groups, with $\mathrm{pK}_{\mathrm{a} 1}=9.6$ and $\mathrm{pK}_{\mathrm{a} 2}=11.3$; these groups are sensitive to $\mathrm{pH}$ variation, act as the active sites for adsorption on the surface of AgCl-NPs, and cause the aggregation of NPs. Therefore, the effect of $\mathrm{pH}$ on the interaction between silver chloride nanoparticles and BPA was studied at different $\mathrm{pH}$ values, ranging from 3.0 to 11 as it is shown in ESM Fig. S5. It is clear from this figure that the SPR band intensity of aggregated AgCl-NPs enlarged significantly with an increase in $\mathrm{pH}$ from neutral to alkaline medium and showed maximum intensity at $\mathrm{pH} 10.5$ with addition of BPA. With further increases in $\mathrm{pH}$ until 11, the SPR band intensity of aggregated AgCl-NPs showed no significant increase (ESM Fig. S4a, b); hence, $\mathrm{pH}$ corresponding to 10.5 has been selected as optimum ones for our study. To confirm the formation of aggregated form of nanoparticles, HR-TEM image and DLS were employed to examine the size change of $\mathrm{AgCl}$ NPs. As displayed in Fig. 2b, the mean hydration size of the as-prepared AgCl-NPs was $65 \mathrm{~nm}$. With addition of BPA, the size increased to about $150 \mathrm{~nm}$ (Fig. 2d), which indicates the formation of aggregated nanoparticles. On the other hand, the HR-TEM image (Fig. 2c) shows that the AgCl-NPs aggregate after adding BPA which results in broader size of NPs; thus, yellow-to-purple color change of solution can be a strong evidence of this aggregation.

In order to get more information about the $\mathrm{pH}$ optimization, the XRD spectrum of AgCl-NPs after interaction with BPA has been obtained in highly alkaline media $(\mathrm{pH}>10.5)$. As we

Fig. 1 Proposed BPA sensing mechanism using green synthesized AgCl-NPs

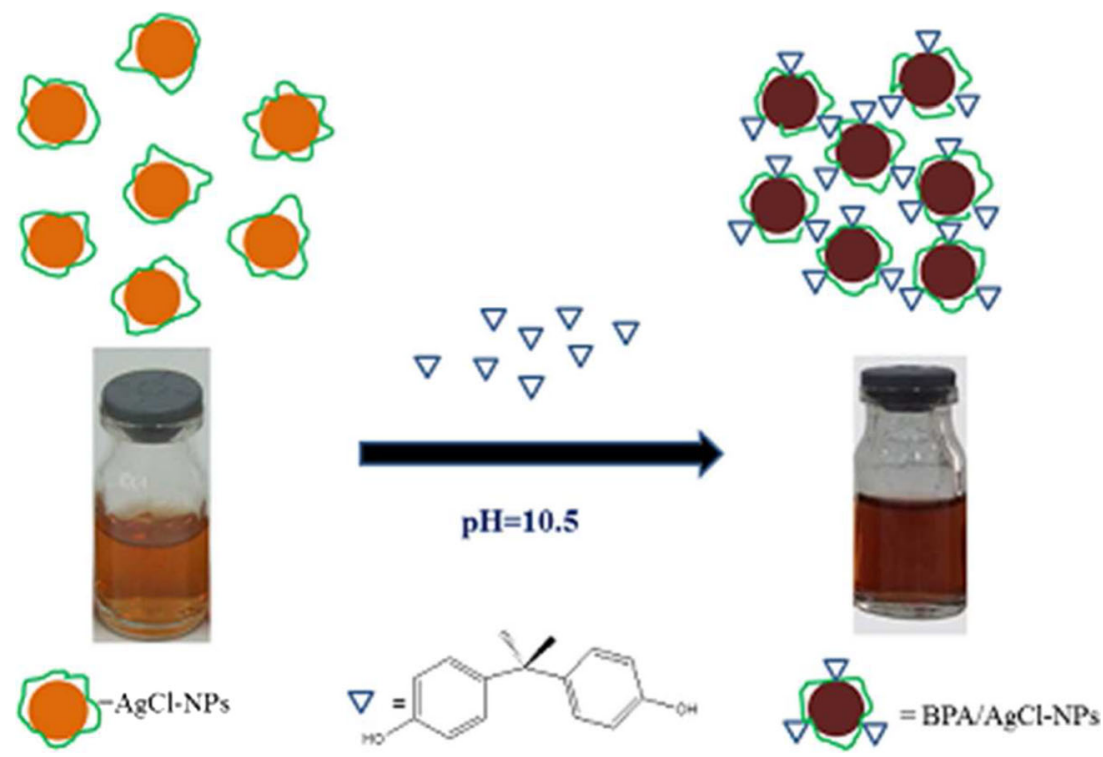


can see from Fig. 2e, silver chloride indicates the representative peak especially the strong peak at 32.2 which was coincident with the $\mathrm{AgCl}\left[\begin{array}{lll}2 & 0 & 0\end{array}\right]$ layer (Fig. 2e) [41]. After interaction of AgCl-NPs with BPA, in higher alkaline media, the new peak at 38.1 is appeared which is related to cubic phase of Ag [ $\left.\begin{array}{lll}1 & 1 & 1\end{array}\right]$ [42]. In other word, at highly alkaline media, the outer silver ions $\left(\mathrm{Ag}^{+}\right)$on surface of silver chloride nanoparticle can reduce into silver $\left(\mathrm{Ag}^{0}\right)$ and led to reduce the reactivity of the silver chloride nanoparticles. Therefore, it was found that highly alkaline media result in production of silver chloride/silver nanostructured materials [40].

For investigating the more details of $\mathrm{AgCl}-\mathrm{NP}$ colorimetric behavior and its priority with respect to AgNPs, we performed a parallel study including the interaction of AgNPs with BPA at different $\mathrm{pH}$ (acidic, neutral, and alkaline) so that AgNP was synthesized using citrate tri-sodium as the conventional chemical reducing agent according to reference [23]. Hence, $50.0 \mathrm{ml}$ of the solution containing $1.0 \mathrm{mM} \mathrm{AgNO}_{3}$ was added into a $100-\mathrm{ml}$ conical flask, and then heated until boiling under continuous stirring. Subsequently, $2.0 \mathrm{ml}$ of $1 \%(w / w)$ citrate tri-sodium was added gradually. The colloidal solution was kept in boiling conditions for $30 \mathrm{~min}$ under vigorous stirring, and then, it was cooled down to room temperature. The color of the above aqueous mixture changed from yellow to brown which demonstrates the formation of AgNPs. Typical experiment including $4 \mathrm{ml}$ of as-prepared AgNPs and $0.5 \mathrm{ml}$ of BPA solutions (correspond to $800 \mu \mathrm{M}$ ) was mixed in the three $\mathrm{pH}$ conditions (acidic, neutral, and alkaline) separately. The results (ESM Fig. S6) were interesting because the SPR band of AgNPs was not changed in the neutral and alkaline conditions which confirmed that no interactions existed between AgNPs and BPA. It should be mentioned that as-prepared AgNPs synthesized using trisoudum citrate has no stability in acidic media (ESM Fig. S5b).

On the other hand, as it is clear from the chemical structure of BPA, there are two acidic hydrogens which can be separated at different $\mathrm{pH}$. Our studied range of $\mathrm{pH}$ is completely corresponding to the range of $\mathrm{pK}_{\mathrm{a}} \mathrm{s}$. In order to get more information about the charge of nanoparticle aggregation in the presence of BPA, the charge of AgCl-NP surface was measured, which is positive in acidic media and highly negative ( $\zeta$-potential of silver chloride nanoparticles ranges from +22.2 to $-50.6 \mathrm{mV}$ at $\mathrm{pH} \mathrm{3-11}$ ) in alkaline solution (ESM Fig. S7). This means that the repulsive interaction between the negatively charged oxygen-containing groups of polyphenols and polysaccharides can be responsible for stability of synthesized nanoparticles in alkaline solution. In the presence of $\mathrm{BPA}$, at $\mathrm{pH}=10.5$, the value of $\zeta$-potential of $\mathrm{AgCl}-\mathrm{NPs}$ increases clearly to the value of -18.6 . This reduction of zeta potential indicates that the repulsive interaction between the negatively charged BPAs and sensors decreases, which led to adsorption of BPA on the surface of AgCl-NPs in this alkaline solutions.
It should be mention that the oxygens of BPA can be good candidates for chemical interaction with silver in silver chloride nanoparticles at optimum $\mathrm{pH}$; however, in order to obtain more information of the possible interactions and their nature, the theoretical study was carried out, and its results will be reported in the following section.

\section{Theoretical study of interactions between synthesized AgCl-NPs and BPA}

The prime focus of the theoretical study is on binding energy, frontier molecular orbital energies, and HOMO-LUMO gap. But for gaining deep insight into the strength of interaction, QTAIM and NBO studies were performed for investigated systems. Two states of BPA, neutral state and its negative ion $\left(\mathrm{BPA}^{-1}\right)$, were selected for electronic studies. The reason behind the selection of these two states is to estimate the affinity of $\mathrm{Ag}$ and $\mathrm{AgCl}$ to both BPA states at neutral and alkaline $\mathrm{pHs}$. Since $\mathrm{pK}_{\mathrm{a}}$ values of BPA are approximately 9.6 and 11.3, at the optimum $\mathrm{pH}$ of the present study (10.5), we just considered $(-1)$ charged compound by removing one acidic hydrogen for theoretical studies. In analyzing zero-point vibrational energy (ZPE) correction on metal ion affinity, binding energy $(\Delta E)$ was carried out in both gas and solvent phases for the two BPA states. In ESM Fig. S8, the optimized structure of the studied compound are presented. There are two stable complexes for BPA-AgCl with very close ground-state electronic energies. Thus, we reported both of them in this study, but the similar possibility was not optimum ones in $\mathrm{BPA}^{-1}$ which has two imaginary frequencies.

The data in Table 1 show that the affinity of $\mathrm{AgCl}$ to BPA in both states is more than that of Ag. For example, the estimated gas phase $\Delta E$ of $\mathrm{Ag}$ and $\mathrm{BPA}$ is $+6.526 \mathrm{kcal} / \mathrm{mol}$ compared to -7.530 and $-9.475 \mathrm{kcal} / \mathrm{mol}$ of $\mathrm{AgCl}$ and BPA interactions. Although the estimated binding energies of both $\mathrm{Ag}$ and $\mathrm{AgCl}$ cases are positive in solvent phase, $\mathrm{AgCl}$ interactions sound less positive and slightly more favorable than BPA-Ag. These results confirm the experimental evidences which show that $\mathrm{AgCl}$ nanoparticles have absolutely more affinity to BPA than silver nanoparticles. On the other hands, the binding energy calculation of $(-1)$ charged BPA shows interesting trend. The $\Delta E$ of $\mathrm{BPA}^{-1}-\mathrm{Ag}$ is $-0.941 \mathrm{kcal} / \mathrm{mol}$ in gas phase, while $\mathrm{BPA}^{-1}-\mathrm{AgCl}$ shows strong binding interaction with $\Delta E=-45.556 \mathrm{kcal} / \mathrm{mol}$. Whereas, in the solvent-phase study, the significant difference between binding energies from $+0.502 \mathrm{kcal} / \mathrm{mol}$ in $\mathrm{BPA}^{-1}-\mathrm{Ag}$ to $-25.289 \mathrm{kcal} / \mathrm{mol}$ in $\mathrm{AgCl}$ case is seen. This finding is also in agreement with experimental evidence which shows really fast detection of BPA in $\mathrm{pH}$ 10.5 by $\mathrm{AgCl}$ nanoparticles while no change is observed by silver nanoparticles.

The frontier molecular orbital (FMO) energies of the investigated compounds are reported in Table S1 in the ESM. From the results, it is obvious that both HOMO and LUMO states in 
a

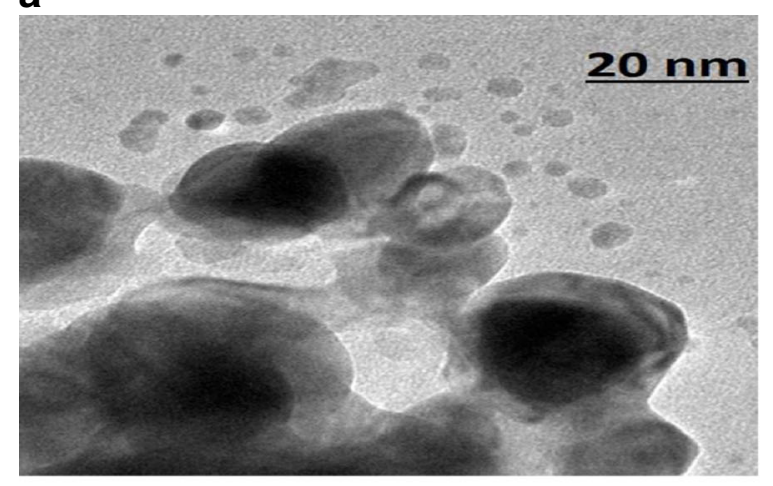

C

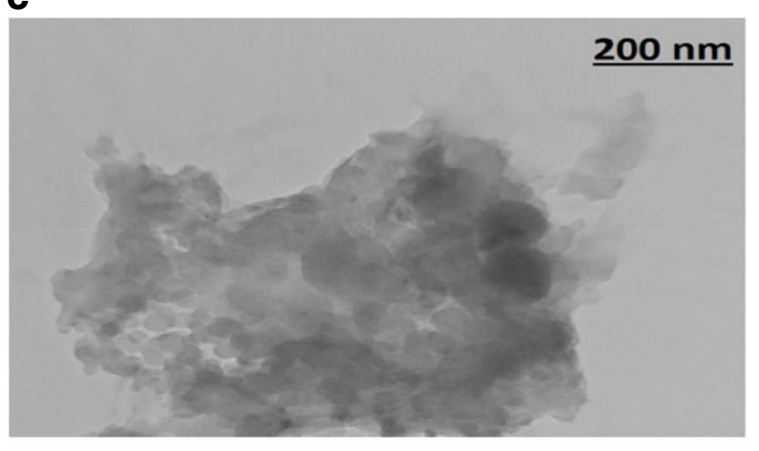

b

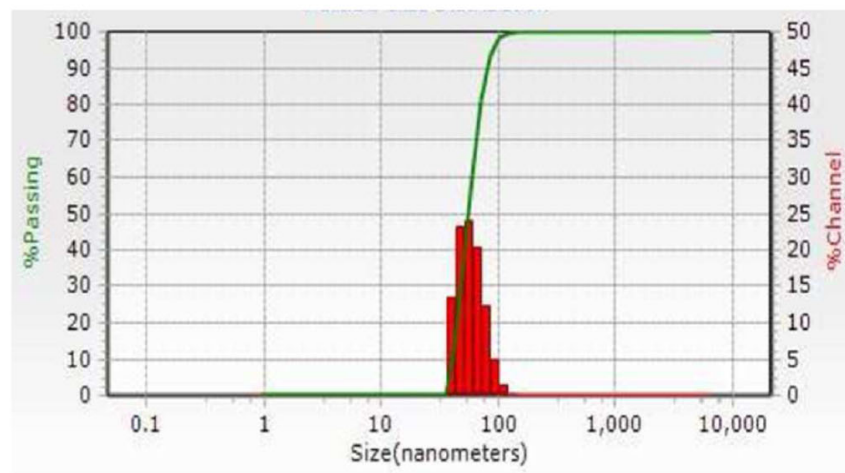

d

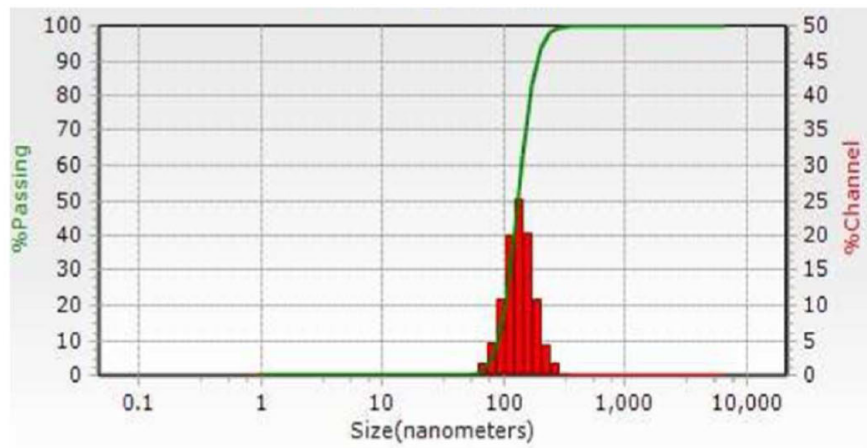

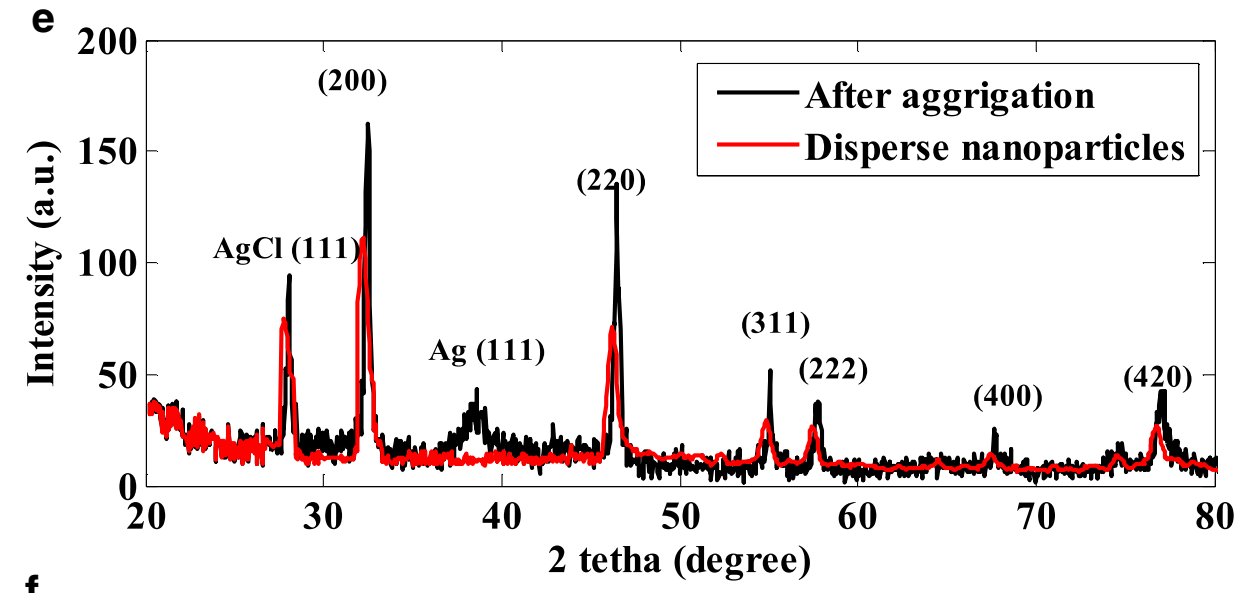

f

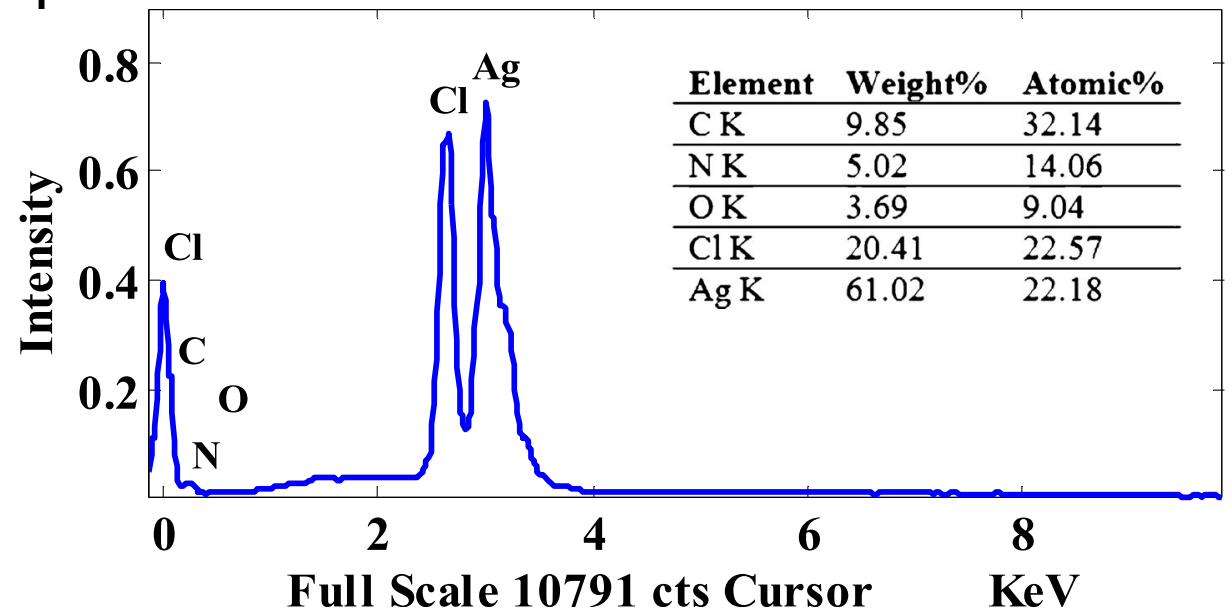


Fig. 2 HR-TEM images of silver chloride nanoparticles solution using $S$. boveanum at various magnifications. a Bare AgCl-NPs. b Dynamic light scattering of dispersed nanoparticles. c AgCl-NPs aggregated when BPA was added. $\mathbf{d}$ Dynamic light scattering of aggregated nanoparticles. $\mathbf{e}$ XRD pattern peaks of disperse and aggregated silver chloride nanoparticles. f Energy-dispersive X-ray analysis of AgCl-NPs synthesized using $S$. boveanum alga

Table 1 The binding energies corrected with zero point vibrational energies at B3lyp level of theory. Energies are in $\mathrm{kcal} / \mathrm{mol}$

\begin{tabular}{lll}
\hline Complex & $\Delta E$ Gas phase & $\Delta E$ Solvent (water) \\
\hline BPA-Ag & +6.526 & +20.958 \\
BPA $^{-1}-\mathrm{Ag}$ & -0.941 & +0.502 \\
BPA-AgCl (1) & -9.475 & +3.451 \\
BPA-AgCl (2) $_{\text {BPA }^{-1}-\mathrm{AgCl}}$ & -7.530 & +13.303 \\
\hline
\end{tabular}

BPA- $\mathrm{AgCl}$ become more stable than those in $\mathrm{Ag}$ complex. By comparing the energy of FMO in investigated complexes with those in $\mathrm{BPA}$, it is evident that $\mathrm{AgCl}$ interactions stabilize the frontier molecular orbitals; however, the HOMO-LUMO gaps become slightly less than free BPA. In contrast, Ag interaction destabilizes HOMO state and decreases the HOMO-LUMO gaps more than that in $\mathrm{AgCl}$. This finding confirms that the complex of $\mathrm{Ag}$ and $\mathrm{BPA}$ is more unstable than that of $\mathrm{AgCl}$ due to its more chemical reactivity/lower HOMO-LUMO gap. Furthermore, the solvation model also shows similar trends to gas phase. The trend of FMO energies in $\mathrm{Ag}$ and $\mathrm{AgCl}$ complexation with $\mathrm{BPA}^{-1}$ is also similar to that in BPA complexes, and $\mathrm{Ag}$ interaction leads to more unstable HOMO state and less HOMO-LUMO gab consequently, which can be an evidence for unstable complex of $\mathrm{BPA}^{-1}-\mathrm{Ag}$.

Furthermore, bond critical point (BCP) corresponds to the minimum of the electron density along the bond path of an atom pair, which the chemical bonding interactions may be characterized according to the properties of electron and energy densities. QTAIM study of investigated system reveals that the values of all $\rho_{B C}$ are less than 0.10 a.u. which confirm the closed shell interactions [43]. As it is reported in Table 2, the bond critical point densities of $\mathrm{AgCl}$ interactions with BPA in both anionic and neutral states $\left(\rho_{B C}=0.073\right.$ a.u. in $\mathrm{BPA}^{-1}$ and $\rho_{B C}=0.050$ a.u. in BPA) are more than those of $\mathrm{Ag}$ $\left(\rho_{B C}=0.031\right.$ a.u. in $\mathrm{BPA}^{-1}$ and $\rho_{B C}=0.004$ a.u. in BPA). It is obvious from the results in Table 2 that the values of all $\rho_{B C}$ are less than 0.10 a.u. which mainly confirm the closed shell interactions. Furthermore, the Laplacian at the BCP, $\nabla^{2} \rho$, is the sum of the three curvatures of the density at the bond critical point $\left(\lambda_{1}, \lambda_{2}\right.$, and $\left.\lambda_{3}\right)$; the first two ones which are perpendicular to bond baths are always negative but the latter one, lying along bond path, is positive. The overall positive values of $\nabla^{2} \rho$ illustrate the extent that density is depleted in the region of the interatomic surface and consequently concentrated in the individual atomic basins while the $\nabla^{2} \rho$ shows the density is concentrated along bond path. Total electronic energy density is another important parameter which is evaluated in accordance with following formula:

$H_{B C}=V_{B C}+G_{B C}$,

where $V_{B C}$ is potential energy density (virial field) and $G_{B C}$ is gradient kinetic energy density. When Laplacian $\nabla^{2} \rho$ and $\mathrm{H}$ are both positive, the bond is electrostatic (closed shell), or if both are negative, the bond should be covalent. There is another possibility which is the case that $\nabla^{2} \rho$ is positive and $\mathrm{H}(\mathrm{r})$ is negative, so that the interaction is called partial electrostatic and partial covalent [44]. In this study, the natures of interactions in both $\mathrm{BPA}^{-1}$ complexes are partially electrostatic and covalent because of positive $\nabla^{2} \rho$ and negative value of $\mathrm{H}(\mathrm{r})$. Moreover, the BPA-Ag and BPA-AgCl (2) show completely electrostatic interaction. There is only one possibility for partially covalent/ electrostatic interaction between $\mathrm{AgCl}$ and BPA which is corresponding to $\mathrm{BPA}-\mathrm{AgCl}$ (1). This finding indicates that the relatively strong electrostatic interaction exists between $\mathrm{AgCl}$ and BPA and its anion, which has also partially covalent character in

Table 2 The QTAIM parameters at bond critical point of Ag interactions with BPA and its anion. (All units are in a.u.)

\begin{tabular}{|c|c|c|c|c|c|}
\hline Complex & Interaction & $\rho(r)$ & $\nabla^{2} \rho(r)$ & $H(r)$ & Nature of interaction \\
\hline BPA-Ag & Ag-Ring & 0.004 & 0.008 & $0<+0.000$ & Electrostatic \\
\hline $\mathrm{BPA}^{-1}-\mathrm{Ag}$ & Ag-Ring & 0.031 & 0.083 & -0.002 & $\begin{array}{l}\text { Partial } \\
\quad \text { covalent/electrostatic }\end{array}$ \\
\hline $\begin{array}{l}\mathrm{BPA}-\mathrm{AgCl} \\
\text { (1) }\end{array}$ & $\mathrm{Ag}-\mathrm{O}_{30}$ & 0.050 & 0.275 & $0>-0.000$ & $\begin{array}{l}\text { Partial } \\
\quad \text { covalent/electrostatic }\end{array}$ \\
\hline BPA-AgCl(2) & Ag-Ring & 0.007 & 0.027 & +0.001 & Electrostatic \\
\hline $\mathrm{BPA}^{-1}-\mathrm{AgCl}$ & $\mathrm{Ag}-\mathrm{O}_{30}$ & 0.073 & 0.394 & -0.005 & $\begin{array}{l}\text { Partial } \\
\quad \text { covalent/electrostatic }\end{array}$ \\
\hline
\end{tabular}


$\mathrm{BPA}^{-1}$ complexes. Finally, there is low charge density at bond critical point in $\mathrm{Ag}$ atom interaction with BPA but this value increases to 0.031 in $\mathrm{BPA}^{-1}-\mathrm{Ag}$.

A second-order perturbation theory analysis of the Fock matrix was carried out to evaluate the donoracceptor interaction in the NBO basis. The interactions result in a loss of occupancy from the localized NBOs of the idealized Lewis structure into the empty nonLewis orbitals. For each donor $\mathrm{NBO}(i)$ and acceptor $(j)$, the stabilization energy $E^{(2)}$ associated with the delocalization $i \rightarrow j$ is estimated as follows:

$E^{(2)}=\Delta E_{i j}=\Delta E_{C T}=-2 \frac{\left\langle i\left|F^{\wedge}\right| j\right\rangle^{2}}{\varepsilon_{j}-\varepsilon_{i}}$,

where $\varepsilon_{i}$ and $\varepsilon_{j}$ are NBO orbital energies, and $F^{\wedge}$ is the Fock operator.

The quantities of transferred charge from a given donor orbital to a given acceptor orbital may be estimated again using the perturbation theory arguments, leading to the following approximate formula:

$q_{C T} \approx 2\left(\frac{\left\langle i\left|F^{\wedge}\right| j\right\rangle}{\varepsilon_{j}-\varepsilon_{i}}\right)^{2}$.

The NBO charge transfer study shows that there is $26.34 \mathrm{kcal} / \mathrm{mol}$ energy due to charge transfer from lone pairs of oxygen in BPA to $\mathrm{Lp}^{*} \mathrm{Ag}$ in $\mathrm{AgCl}$ (2) but there is no charge transfer between BPA and Ag atom (ESM Table S2). In $\mathrm{BPA}^{-1}$, the similar trend is seen, and $55.99 \mathrm{kcal} / \mathrm{mol}$ resulted in charge delocalization from lone pairs of oxygen to $\mathrm{Lp} * \mathrm{Ag}$ in $\mathrm{AgCl}$ while there is no significant charge transfer from $\mathrm{BPA}^{-1}$ to $\mathrm{Ag}$ atom again. The quantity of charge transferred during the interaction in $\mathrm{BPA}^{-1}$ is also more than that in BPA. Although, in BPA-AgCl (2), there is one significant charge transfer due to $\pi_{\mathrm{C} 14-\mathrm{C} 17} \rightarrow \mathrm{Lp}^{*}(6)$ of $\mathrm{Ag}$, this shows that both possibilities may be important during interaction of $\mathrm{AgCl}$ with
Fig. 3 The possible chemical interactions between BPA and $\mathrm{AgCl}$ at the alkaline and neutral mediums

\section{Possiblity 1 in neutral solution}
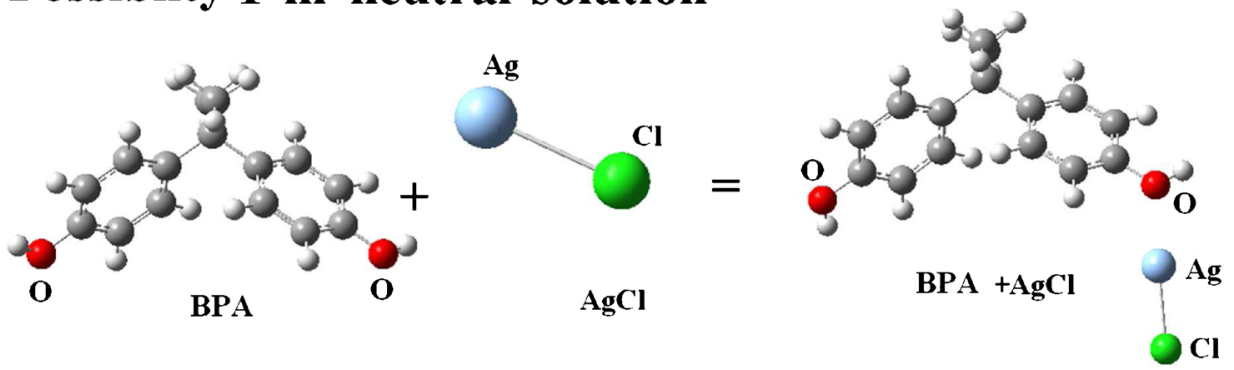

Possiblity 2 in neutral solution
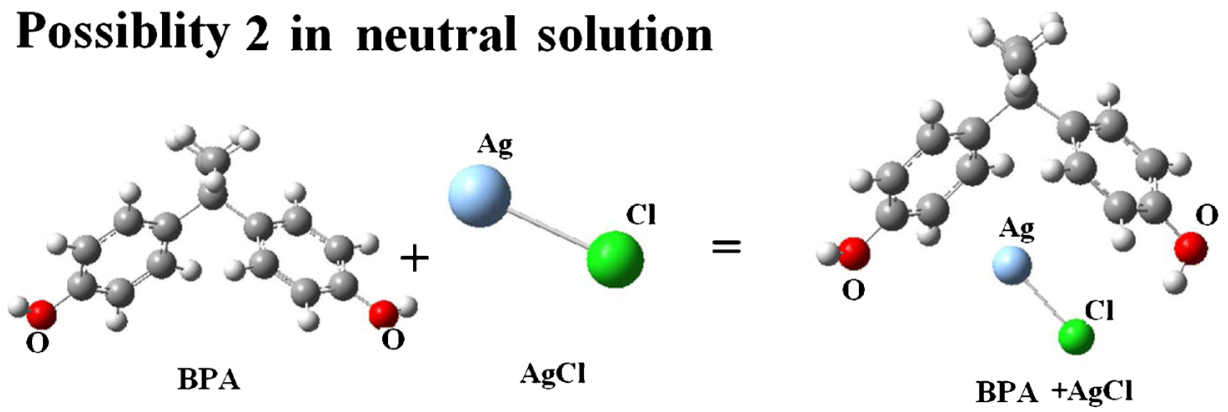

\section{In alkaline solution}
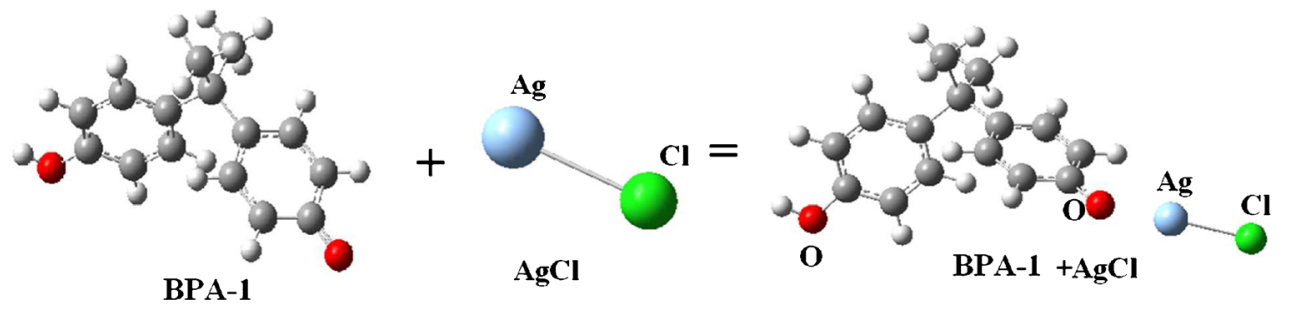
BPA which give stronger stabilization to the structure. In conclusion, the larger the $E^{(2)}$ value, the more intensive is the interaction between electron donors and $\mathrm{AgCl}$.

Finally, in accordance with the theoretical results, the chemical interactions between AgCl-NPs and BPA can be simulated at the molecular level through two possibilities which are also likely to be generalized at nanoscale. These two are illustrated in Fig. 3 schematically. Although two possibilities have close binding energies (Table 1), with increase of $\mathrm{pH}$, the first one becomes more favor and superiority of $\mathrm{AgCl}-\mathrm{O}$ interaction turns out obviously. It can be concluded that at alkaline medium, the BPA well interacts with the $\mathrm{AgCl}$ molecules from its oxygens. These relatively strong electrostatic/covalence interactions can aid to attract more BPAs to the AgCl-NPs particularly those accessible molecules on the surfaces of nanoparticles.
Fig. 4 Colorimetric sensing of BPA using AgCl-NPs. a Photographs of AgCl-NP solutions with different BPA concentrations. b Extinction spectra of the solutions. c Response $\left(\mathrm{Ex}_{542}\right)$ of the colorimetric assay against increasing BPA concentrations a

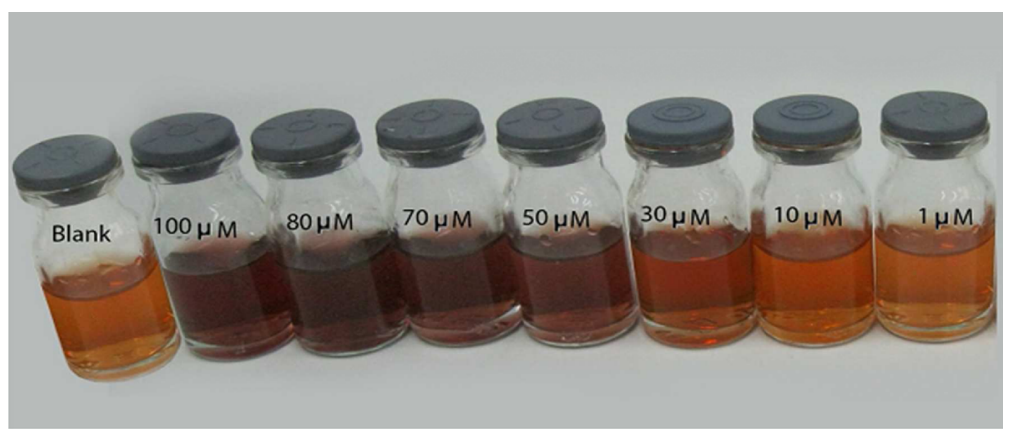

b

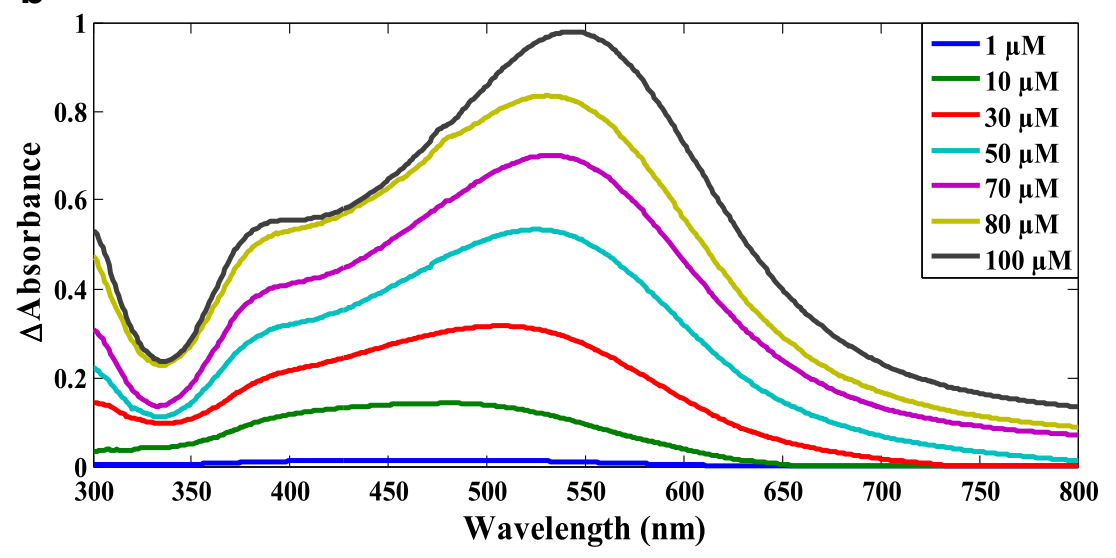

C

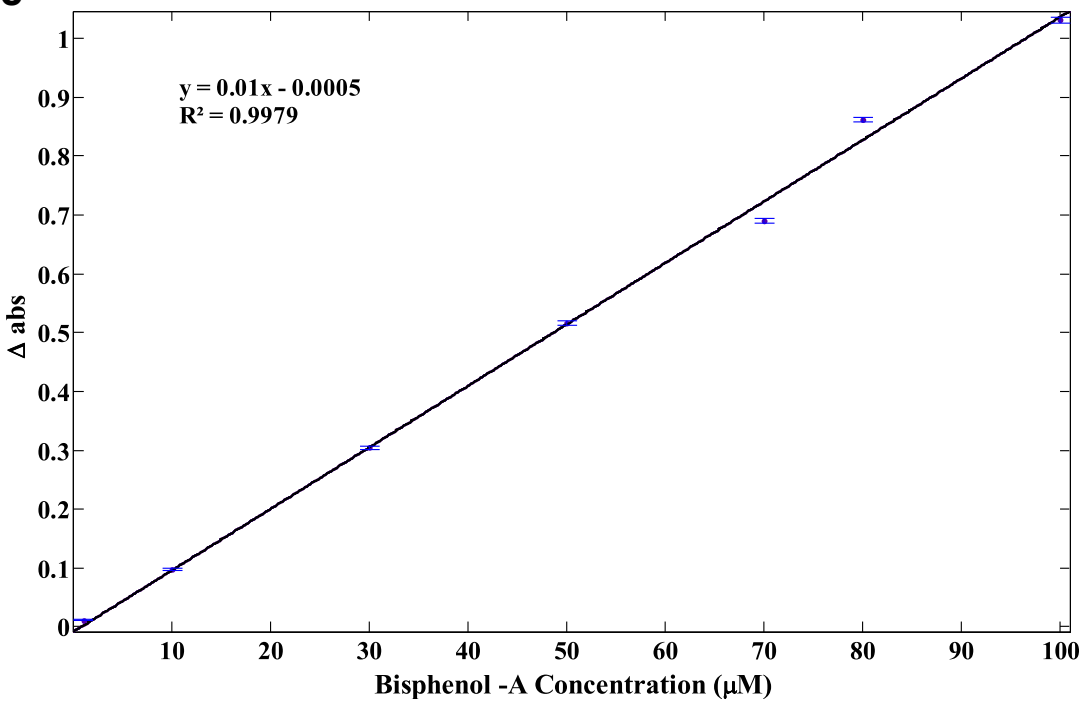


Table 3 Results of the BPA recovery experiments performed in mineral and tap water samples

\begin{tabular}{llll}
\hline & Added $(\mu \mathrm{M})$ & Found $(\mu \mathrm{M})$ & Recovery $(\%)$ \\
\hline Tap water & 5.00 & 5.15 & $103.00 \pm 2.40$ \\
& 10.00 & 9.85 & $98.00 \pm 1.95$ \\
& 15.00 & 14.40 & $96.00 \pm 2.20$ \\
Mineral water & 4.00 & 4.10 & $102.00 \pm 1.20$ \\
& 8.00 & 7.80 & $98.50 \pm 2.50$ \\
& 12.00 & 11.60 & $97.30 \pm 2.30$ \\
\hline
\end{tabular}

\section{Colorimetric detection of bisphenol-A}

To determine the sensitivity of the colorimetric assay, we examined several BPA solutions which had concentrations between 1 and $100 \mu \mathrm{M}$. As the concentration of the BPA increases, the color of the as-prepared AgCl-NPs gradually changes from yellow to purple (Fig. 4a), suggesting the BPA concentration-dependent aggregation of AgCl-NPs. The UVvis spectra of solutions (Fig. 4b) reveal that with increasing BPA concentration, extinction coefficient at $417 \mathrm{~nm}$ gradually increased, and the maximal absorption peak was obviously red shift from around 500 to $542 \mathrm{~nm}$, which also indicates gradually increasing aggregation of the particles. Therefore, the absorbance at 542 was used to quantify the color of the system. A low value was associated with dispersed AgCl-NPs and yellow color while high value is corresponding with aggregated particle and purple color. Figure $4 \mathrm{c}$ shows the concentration-dependent colorimetric response ( $\mathrm{Ex}_{542}$ values) of the assay. In the BPA concentration, range between 1 to $100 \mu \mathrm{M}, \mathrm{Ex}_{542}$ values regularly increase. Further increasing the BPA concentration up to $100 \mu \mathrm{M}$ only increased the colorimetric response slightly (ESM Fig. S9). The analytical response of the assay is highly linear, with a linear regression correlation coefficient of 0.997 at the BPA concentration range of 1 to $100 \mu \mathrm{M}$.

\section{Colorimetric sensing of bisphenol-A in deionized, mineral, and tap water}

In order to detect BPA using synthesized AgCl-NPs in a typical experiment, $4 \mathrm{ml}$ of AgCl-NPs was mixed with $0.5 \mathrm{ml}$ of BPA solutions (in deionized or tap water) at different concentrations to give final BPA concentrations in the range of 1 to $100 \mu \mathrm{M}$. Then, the $\mathrm{pH}$ of this solution was set at suitable value $(\mathrm{pH}=10.5)$. Finally, the color changes were detected by the naked eye and followed by recording the UV-vis absorption spectra so that the photographs were taken with a digital camera (12.0 megapixels), and the absorption measurements were performed using an UV-vis spectrophotometer in the range of 300 to $800 \mathrm{~nm}$. The analytical responses were calculated by extinction coefficient of the AgCl-NP suspension at $542 \mathrm{~nm}$ $\left(\mathrm{Ex}_{542}\right)$. All experiments were performed in triplicate. The limit of detection (LOD) values for detection of BPA was calculated by the following formula:

limit of detectable response

$=$ average response of the blank

$+(3 \times$ standard deviation of the blank $)$.

Finally, the limit of detection (lowest detectable) BPA concentration was calculated to be $45 \mathrm{nM}$ by our assay.

To evaluate the practical applications of the colorimetric assay to real samples, tap and mineral water (sampled from Bushehr, south of Iran) was spiked with BPA $(5,10$, and $15 \mu \mathrm{M}$ for tap and 4,8 , and $12 \mu \mathrm{M}$ for mineral water) and tested. The obtained results of recovery experiments using spiked BPA in tap and mineral water samples, are reported in Table 3. We observed an average recovery value of $99.0 \%$ for tab water which indicates that our colorimetric assay can be used for the detection of BPA in real samples. The same trend was obtained in the case of mineral water. High recovery percentages and low standard deviation in the experiments indicate the high accuracy of our colorimetric assay.

\section{Selectivity of the colorimetric assay}

To check the selectivity of the colorimetric assay against BPA, common cation, anion, and organic interferences were tested. Figure S10 in the ESM illustrates the color changes against the several variations of possible interference quantities in samples in addition to BPA. This figure displays a distinct color change from yellow to purple only in the presence of BPA, which is according to an intense increase of the absorption intensity at $542 \mathrm{~nm}$ (aggregated form). The other substances (cation, anion, and especially organic materials) had no obvious effect on color change, which proved that our synthesized AgCl-NPs are efficient for BPA detection. This observed selectivity can be related to solubility of chloride products of metal cation interferences. Most of them have higher solubility product than silver chloride. Furthermore, the same reason can be considered for interaction of anions with silver chloride nanoparticles. The solubility of carbonate, fluoride, sulfate, and nitrate of silver are more than that of chloride, and it is sensible to have no interfering.

\section{Conclusion}

The present investigation used the simple and green approach for the synthesis of AgCl-NPs using brown marine algae (S. boveanum). The synthesized NPs were characterized by UV-visible, HR-TEM, DLS, EDXA, XRD, and FTIR 
techniques. The assay uses marine algae aqueous extract acted as both chlorine source and capping agents for the stabilization of AgCl-NPs. Moreover, a simple and rapid colorimetric assay for BPA detection in tap and mineral water was developed by synthesized AgCl-NPs for the first time. Optimum $\mathrm{pH}$ for the interaction of BPA and AgCl-NPs was 10.5. In this condition, the oxygen of BPA plays an important role to attract silver chloride NPs. The theoretical study of interactions at DFT level of theory confirmed this interaction too. Furthermore, preferred affinity of AgCl-NPs towards BPA with respect to $\mathrm{AgNPs}$ was illustrated by binding free energies which shows that AgCl-NPs have larger affinity than AgNPs. The detection limit of the colorimetric assay is around $45 \mathrm{nM}$ at optimum condition. Also, the selectivity of our sensor was demonstrated in the presence of several other heavy metal ions, anions, and organic substances at high concentrations $(200 \mu \mathrm{M})$. In conclusion, our simple assay is one of the most simple, sensitive, and rapid colorimetric BPA sensors compared with many conventional instrument methods. However, the ultimate goal of this colorimetric assay is the application of it in the simple and simultaneously detection of bisphenol series. This will be done using combination of colorimetric assay with efficient chemometrics method which is under study in our research group. Finally, green synthesis of AgCl-NPs and its colorimetric assay sensor will be helpful in providing an insight into the development of colorimetric agents in the near future.

\section{Compliance with ethical standards}

Conflict of interest The authors declare that they have no conflicts of interest.

\section{References}

1. Mayer KM, Hafner JH. Localized surface plasmon resonance sensors. Chem Rev. 2011;111(6):3828-57.

2. Wang J, Zhang P, Li JY, Chen LQ, Huang CZ, Li YF. Adenosineaptamer recognition-induced assembly of gold nanorods and a highly sensitive plasmon resonance coupling assay of adenosine in the brain of model SD rat. Analyst. 2010;135(11):2826-31.

3. Zhou W, Gao X, Liu D, Chen X. Gold nanoparticles for in vitro diagnostics. Chem Rev. 2015;115(19):10575-636.

4. Zhang H, Jin M, Xiong Y, Lim B, Xia Y. Shape-controlled synthesis of Pd nanocrystals and their catalytic applications. Accounts Chem Res. 2012;46(8):1783-94.

5. Yang Y, Wang X, Cui Q, Cao Q, Li L. Self-assembly of fluorescent organic nanoparticles for iron (III) sensing and cellular imaging. ACS Appl Mater Interfaces. 2016;8(11):7740-8.

6. Guo X-m WZ, Li W, Wang Z-h, et al. Appropriate size of magnetic nanoparticles for various bio-applications in cancer diagnostics and therapy. ACS Appl Mater Interfaces. 2016;8(5):3092-106.

7. Chen L, Li H, He H, Wu H, Jin Y. Smart plasmonic glucose nanosensors as generic theranostic agents for targeting-free cancer cell screening and killing. Anal Chem. 2015;87(13):6868-74.
8. Jana NR, Gearheart L, Murphy CJ. Wet chemical synthesis of silver nanorods and nanowires of controllable aspect ratio. Electronic supplementary information (ESI) available: UV-VIS spectra of silver nanorods. Chem Commun. 2001;7:617-8.

9. Hanada N, Ichikawa T, Fujii H. Catalytic effect of nanoparticle 3dtransition metals on hydrogen storage properties in magnesium hydride $\mathrm{MgH}_{2}$ prepared by mechanical milling. J Phys Chem B. 2005;109(15):7188-94.

10. Zhang Y, Yuan X, Wang Y, Chen Y. One-pot photochemical synthesis of graphene composites uniformly deposited with silver nanoparticles and their high catalytic activity towards the reduction of 2-nitroaniline. J Mater Chem. 2012;22(15):7245-51.

11. Yamauchi Y, Tonegawa A, Komatsu M, et al. Electrochemical synthesis of mesoporous $\mathrm{Pt}-\mathrm{Au}$ binary alloys with tunable compositions for enhancement of electrochemical performance. J Am Chem Soc. 2012;134(11):5100-9.

12. Sener G, Uzun L, Denizli A. Lysine-promoted colorimetric response of gold nanoparticles: a simple assay for ultrasensitive mercury (II) detection. Anal Chem. 2013;86(1):514-20.

13. Chen S, Fang Y-M, Xiao Q, et al. Rapid visual detection of aluminium ion using citrate capped gold nanoparticles. Analyst. 2012;137(9):2021-3.

14. Kundu S, Lau S, Liang H. Shape-controlled catalysis by cetyltrimethylammonium bromide terminated gold nanospheres, nanorods, and nanoprisms. J Phys Chem C. 2009;113(13):5150-6.

15. Chen Z, Zhang X, Cao H, Huang Y. Chitosan-capped silver nanoparticles as a highly selective colorimetric probe for visual detection of aromatic ortho-trihydroxy phenols. Analyst. 2013;138(8):23439.

16. Kumar S, Gradzielski M, Mehta S. The critical role of surfactants towards CdS nanoparticles: synthesis, stability, optical and PL emission properties. RSC Adv. 2013;3(8):2662-76.

17. Alkilany AM, Nagaria PK, Hexel CR, Shaw TJ, Murphy CJ, Wyatt MD. Cellular uptake and cytotoxicity of gold nanorods: molecular origin of cytotoxicity and surface effects. Small. 2009;5(6):701-8.

18. Annadhasan M, Muthukumarasamyvel T, Sankar Babu V, Rajendiran N. Green synthesized silver and gold nanoparticles for colorimetric detection of $\mathrm{Hg}^{2+}, \mathrm{Pb}^{2+}$, and $\mathrm{Mn}^{2+}$ in aqueous medium. ACS Sustainable Chem Eng. 2014;2(4):887-96.

19. Iravani S. Green synthesis of metal nanoparticles using plants. Green Chem. 2011;13(10):2638-50.

20. Raveendran P, Fu J, Wallen SL. Completely "green" synthesis and stabilization of metal nanoparticles. J Am Chem Soc. 2003;125(46):13940-1.

21. Azizi S, Ahmad MB, Namvar F, Mohamad R. Green biosynthesis and characterization of zinc oxide nanoparticles using brown marine macroalga Sargassum muticum aqueous extract. Mater letts. 2014;116:275-7.

22. Dhas TS, Kumar VG, Karthick V, Angel KJ, Govindaraju K. Facile synthesis of silver chloride nanoparticles using marine alga and its antibacterial efficacy. Spectrochim Acta A. 2014;120:416-20.

23. Huang JT, Yang XX, Zeng QL, Wang J. A simple green route to prepare stable silver nanoparticles with pear juice and a new selective colorimetric method for detection of cysteine. Analyst. 2013;138(18):5296-302.

24. Rajeshkumar S, Kannan C, Annadurai G. Green synthesis of silver nanoparticles using marine brown algae Turbinaria conoides and its antibacterial activity. Int J Pharm Bio Sci. 2012;3(4):502-10.

25. Chen X, Wang C, Tan X, Wang J. Determination of bisphenol A in water via inhibition of silver nanoparticles-enhanced chemiluminescence. Anal Chim Acta. 2011;689(1):92-6.

26. Muñoz-de-Toro M, Markey CM, Wadia PR, et al. Perinatal exposure to bisphenol-A alters peripubertal mammary gland development in mice. Endocrinology. 2005;146(9):4138-47.

27. Becke AD. Density-functional thermochemistry. III The role of exact exchange. J Chem Phys. 1993;98(7):5648-52. 
28. Lee C, Yang W, Parr RG. Development of the Colle-Salvetti correlation-energy formula into a functional of the electron density. Phys Rev B. 1988;37(2):785.

29. Glendening E, Reed A, Carpenter J, Weinhold F. NBO Version 3.1, TCI, vol. 65. Madison: University of Wisconsin; 1998.

30. Bader RF. Atoms in molecules. Wiley Online Library. 1990.

31. Foster J, Weinhold F. Natural hybrid orbitals. J Am Chem Soc. 1980;102(24):7211-8.

32. Reed AE, Weinstock RB, Weinhold F. Natural population analysis. J Chem Phys. 1985;83(2):735-46.

33. Biegler-König F, Schönbohm J. Update of the AIM2000 - program for atoms in molecules. J Comput Chem. 2002;23(15):1489-94.

34. Frisch M, Trucks G, Schlegel HB, et al. Gaussian 09, revision A. 02, vol. 19. Wallingford, CT: Gaussian, Inc; 2009. p. 227-38.

35. Gopinath V, Priyadarshini S, Priyadharsshini NM, Pandian K, Velusamy P. Biogenic synthesis of antibacterial silver chloride nanoparticles using leaf extracts of Cissus quadrangularis Linn. Mater Letts. 2013;91:224-7.

36. Mulvaney P. Surface plasmon spectroscopy of nanosized metal particles. Langmuir. 1996;12(3):788-800.

37. Fayer MD. Ultrafast infrared vibrational spectroscopy. CRC Press. 2013.
38. Rajeswari A, Kumar VG, Karthick V, Dhas TS, Potluri SL. Hydrothermal synthesis of hydroxyapatite plates prepared using low molecular weight heparin (LMWH). Colloid Surface B. 2013;111:764-8.

39. Gómez-Ordóñez E, Rupérez P. FTIR-ATR spectroscopy as a tool for polysaccharide identification in edible brown and red seaweeds. Food Hydrocolloid. 2011;25(6):1514-20.

40. Choi M, Shin K-H, Jang J. Plasmonic photocatalytic system using silver chloride/silver nanostructures under visible light. J Colloid Interf Sci. 2010;341(1):83-7.

41. Schürch D, Currao A, Sarkar S, Hodes G, Calzaferri G. The silver chloride photoanode in photoelectrochemical water splitting. J Phys Chem B. 2002;106(49):12764-75.

42. Kakuta N, Goto N, Ohkita H, Mizushima T. Silver bromide as a photocatalyst for hydrogen generation from $\mathrm{CH} 3 \mathrm{OH} / \mathrm{H} 2 \mathrm{O}$ solution. J Phys Chem B. 1999;103(29):5917-9.

43. Bader R, Schleyer PR. Encyclopedia of computational chemistry. Chichester, UK: John Wiley \& Sons, Ltd.; 2002.

44. Bader RF. A bond path: a universal indicator of bonded interactions. J Phys Chem A. 1998;102(37):7314-23. 